\title{
Paracoccidiomicose juvenil: um relato de caso
}

\author{
Juvenile Paracoccidiomycosis mycosis: a case report \\ Paracoccidiomicosis juvenil: reporte de un caso
}

Patrícia Macedo Bernardino ${ }^{*}$, Ana Cláudia Fernandes Azarias ${ }^{1}$, Fernanda Gomes Junqueira dos Santos ${ }^{1}$, Adriano Braga ${ }^{1}$, Wilson Cunha Junior ${ }^{1}$.

\section{RESUMO}

Objetivo: Descrever um caso de paracoccidioidomicose em um paciente previamente hígido, pois se trata de uma patologia ainda subnotificada. Detalhamento do caso: Paciente jovem do sexo masculino iniciou com quadro de lesões de pele e linfonodomegalia generalizada além de febre diária e perda de peso durante duas semanas. Ao exame físico foi evidenciado além das lesões de pele e aumento dos linfonodos uma hepatoesplenomegalia. Iniciado tratamento para PBmicose e paciente entrou em remissão das lesões recebendo alta para acompanhamento ambulatorial. Considerações finais: PBmicose é uma micose profunda de grande importância epidemiológica ainda nos dias de hoje. No entanto a mesma por não ser de notificação compulsória é ainda subdiagnosticada. É uma patologia que deve ser sempre lembrada em casos de linfonodomegalia generalizada e hepatoesplenomegalia. Seu diagnóstico envolve fatores clínicos, laboratoriais e biopsia. Seu tratamento envolve drogas de acesso amplo como o sulfametoxazol + trimetoprim e pode ser realizado em ambiente ambulatorial.

Palavras-chave: Pbmicose, Micoses profundas, Linfonodomegalia Generalizada.

\begin{abstract}
Objective: To describe a case of paracoccidioidomycosis in a previously healthy patient, as it is a pathology that is still underreported. Case details: Young male patient started with skin lesions and generalized lymph node enlargement in addition to daily fever and weight loss for two weeks. Physical examination revealed hepatosplenomegaly in addition to skin lesions and enlarged lymph nodes. Treatment for PBmicose started and patient went into remission of the lesions being discharged for outpatient follow-up. Final considerations: PBmicose is a deep mycosis of great epidemiological importance even today. However, as it is not mandatory, it is still underdiagnosed. It is a pathology that should always be remembered in cases of generalized lymph node enlargement and hepatosplenomegaly. Its diagnosis involves clinical, laboratory and biopsy factors. Its treatment involves drugs of broad access such as sulfamethoxazole + trimethoprim and can be performed in an outpatient setting
\end{abstract}

Keywords: Pbmicose, Deep mycoses, Generalized lymph node enlargement.

\section{RESUMEN}

Objetivo: Describir un caso de paracoccidioidomicosis en un paciente previamente sano, por tratarse de una patología aún infreportada. Detalle del caso: Paciente joven de sexo masculino que inició con lesiones cutáneas y agrandamiento generalizado de los ganglios linfáticos además de fiebre diaria y pérdida de peso. por dos semanas. El examen físico reveló hepatoesplenomegalia además de lesiones cutáneas y agrandamiento de los ganglios linfáticos. Se inició tratamiento para PBmicose y el paciente entró en remisión de las lesiones y fue dado de alta para seguimiento ambulatorio. Consideraciones finales: La PBmicosa es una micosis profunda de gran importancia epidemiológica incluso en la actualidad. Sin embargo, como no es obligatorio, todavía está infradiagnosticado. Es una patología que siempre debe recordarse en casos de agrandamiento ganglionar generalizado y hepatoesplenomegalia. Su diagnóstico involucra factores clínicos, de laboratorio y de biopsia. Su tratamiento implica fármacos de amplio acceso como sulfametoxazol + trimetoprima y puede realizarse de forma ambulatoria.

Palabras clave: Pbmicosa, micosis profundas, agrandamiento generalizado de los ganglios linfáticos.

${ }^{1}$ Fundação Santa Casa de Misericórdia de Franca, Franca - SP. *E-mail: patmber@hotmail.com

SUBMETIDO EM: 11/2020 | ACEITO EM: 12/2020 | PUBLICADO EM: 2/2020 


\section{INTRODUÇÃO}

A Paracoccidioidomicose é uma micose sistêmica de acometimento mucocutâneo, causada pelo fungo do gênero Paracoccidioides spp, sendo as maiores incidências registradas no Brasil, Argentina, Colômbia e Venezuela (SHIKANAI-YASUDA MA, et al., 2017; MESQUITA PMF, et al., 2016). No Brasil é considerada endêmica nos estados de São Paulo, Minas Gerais, Rio de Janeiro, Rio Grande do Sul, Paraná, Mato Grosso e Mato Grosso do Sul. Predominantemente é de incidência rural apesar de nos últimos anos apresentar registros de casos em regiões suburbanas e urbanas (MESQUITA PMF, et al., 2016; MILLINGTON MA, et al., 2018).

O fungo é termodimórfico com destaque a duas espécies patogênicas: Paracoccidioides brasiliensis ( $P$. brasiliensis) e Paracoccidioides lutzii (P.lutzii). Esses estão dispersos no meio ambiente e se espalham pelo solo (SHIKANAI-YASUDA MA, et al., 2017; MESQUITA PMF, et al., 2016; MILLINGTON MA, et al., 2018; ROSARIO JS, et al., 2017). Seu habitat ainda não é determinado, mas é considerado que ele viva como saprófita no solo e/ou vegetais em temperaturas entre 10 e 28 graus e locais úmidos, com invernos curtos e verões chuvosos (ROSARIO JS, et al., 2017; MELO JA, et al, 2017).

Esta não é uma doença de notificação compulsória, mesmo assim, é a oitava causa de morte no Brasil considerando as doenças infecciosas e parasitárias predominantemente crônicas. Como acomete indivíduos em idade produtiva e requer tratamento prolongado afeta a sociedade de forma economicamente já que leva tais pacientes a afastamentos e até a morte (MESQUITA PMF, et al., 2016; GAETTLI-JJE, et al., 2016). Todas as raças parecem ser igualmente suscetíveis. Ocorre cerca de nove vezes mais em homens do que em mulheres o que, segundo a literatura, é consequência das atividades rurais e maior contato com o solo pelos homens já que o contágio se dá principalmente após manipulação do solo (MESQUITA PMF, et al., 2016; SILVA JF, et al., 2016; VALLE ACF, et al., 2017; MACEDO PM, et al., 2017; SHIHIKANAI-YASUDA MA, et al., 2017).

A paracoccidioidomicose foi descrita pela primeira vez, em 1908, por Adolfo Lutz. Posteriormente, provou-se a proximidade filogenética entre o P.brasiliensis e os fungos dos gêneros Histoplasma, Emmonsia e Blastomyces. São considerados hospedeiros definitivos o ser humano, tatu e o cão (MELO JA, et al., 2017; ROSARIO JS, et al., 2017).

A exposição ao fungo está relacionada com o manejo do solo contaminado, como, por exemplo, atividades agrícolas, terraplenagem, preparo de solo, práticas de jardinagens, transporte de produtos vegetais, entre outras. A maioria dos indivíduos que adoeceram apresenta história de atividade agrícola exercida nas duas primeiras décadas de vida. Hábitos de vida como, tabagismo e etilismo, também são considerados fatores de risco frequentemente associados à micose, e ao agravamento do seu quadro clínico (MILLINGTON MA, et al., 2018; MELO JA, et al., 2017).

Não existe transmissão inter-humana do fungo Paracoccidioides spp. No entanto, os indivíduos estão expostos ao risco por inalação de propágulos infectantes dispersos no solo. A principal porta de entrada do fungo no organismo é por via inalatória. Os órgãos comumente afetados são os pulmões (50-100\%), seguidos da pele, mucosas, linfonodos, adrenais, sistema nervoso central, fígado e ossos (MILLINGTON MA, et al., 2018; MELO JA, et al., 2017; ROSARIO JS, et al., 2017). O quadro clínico da Pbmicose é variado, porém alguns achados são constantes: adenopatia localizada ou sistêmica, acometimento da mucosa oral e da pele e do aparelho respiratório e digestivo (MESQUITA PMF, et al., 2016; MACEDO PM, et al., 2016).

Os sintomas gastrointestinais podem ser inespecíficos como dor, diarreia, algumas vezes obstipação. Outros sintomas inespecíficos relatados são fraqueza, emagrecimento, cefaleia, inapetência e febre (MESQUITA PMF, et al., 2016; GAETTLI-JJE, et al., 2016; RADISIC MV, et al., 2017; TOZETTO-MENDOZA TR, et al., 2017). Na mucosa oral geralmente surgem lesões múltiplas pápulo erosivas, ulceradas e hipertróficas, com aspecto granuloso e avermelhado, semelhante à superfície de amora, denominada de estomatite morabiliiforme envolvendo gengiva, mucosas, palato, língua e assoalho bucal. Quando já avançadas podem levar até mesmo a perfuração do palato duro. Pode ocorrer sialorreia abundante e sensação de prurido, dor e ardor, dificultando a alimentação (MESQUITA PMF, et al., 2016; ALMEIDA FA, et al., 2016). 
Quando atinge o trato respiratório ele induz a uma resposta inflamatória com formação de granuloma, que são coleções de tecidos fagocitários mononuclear, podendo sofrer transformações para células epitelioides e gigantes. O granuloma é uma reação de hipersensibilidade tardia e o desenvolvimento dele contém o patógeno e impede a disseminação pelo organismo (ROSARIO JS, et al., 2017; VOGELMEIER CF, et al., 2017).

O objetivo deste presente trabalho é descrever um caso de importância epidemiológica já que se trata de uma patologia infecciosa adquirida geralmente por indivíduos jovens e que pode se tornar sistêmica. Tal patologia não é muito frequente e não existem muitos estudos recentes devido ao seu subdiagnóstico pois não é de notificação compulsória e também inicialmente se apresenta com sintomas muito inespecíficos e seu diagnóstico conclusivo é através de biópsia das lesões ou tecido afetados e seu tratamento é de longo prazo e muitas vezes a cura propriamente dita não é alcançada.

\section{DETALHAMENTO DO CASO}

Homem, 22 anos, residente em área rural dá entrada no hospital com história de lesões ulceradas em região da face nasal e perioral), clavícula, e algumas lesões em antebraço e perna. Refere febre no período. O mesmo referia perda de $4 \mathrm{~kg}$ (peso inicial de $50 \mathrm{~kg}$. Negava uso de drogas e tabagismo. Referia etilismo social e negava morar em região rural ou viagens recentes. Ao exame físico se apresentava em bom estado geral, corado, hidratado, anictérico, acianótico, afebril, eupneico, linfonodomegalia generalizada em cadeias cervical, clavicular, submandibular.

Apresentava lesão morabiliforme em orofaringe esquerda. Aparelho cardíaco e respiratório sem alterações. Abdome flácido, indolor a palpação, ruídos hidroaéreos presente e normofonéticos. Hepatoesplenomegalia há dois centímetros do rebordo costal. Ausência de alterações neurológicas. Foram coletados exames complementares os quais evidenciaram anemia com hemoglobina de 8,5 padrão normocítico e normocrômico, leucócitos sem desvios, mas aumento de eosinófilos $(7 \%$ com valor de referência de 2 a $5 \%$. Plaquetas aumentadas. Eletrólitos dentro da normalidade. INR de 1,25 e sorologias para hepatite B, C e HIV negativas.

Foi então discutido o caso com otorrinolaringologista para biópsia de lesão em região de orofaringe e biópsia de gânglios. Programada biópsia para o quinto dia de internação paciente mantinha um pico febril diário e sem piora do estado geral. Realizada biópsia em centro cirúrgico, mas como quadro bastante sugestivo de Pbmicose iniciado Anfotericina B empiricamente até o resultado da biópsia. O laudo apresentado encontrou fungos em todas as regiões biopsiadas além de que os gânglios retirados apresentavam secreção então foram enviados para cultura onde foi evidenciado crescimento de Escherichia coli sensível a Sulfametoxazol+Trimetropin e associado está medicação ao tratamento.

Paciente no $10^{\circ}$ dia de internação e já com dose acumulada de Anfotericina de $60 \mathrm{mg}$ começa a ficar afebril, diminuição da hepatoesplenomegalia. Recebe alta após $530 \mathrm{mg}$ de dose acumulada de anfotericina e no oitavo dia de Sulfametoxazol+Trimetropin com regressão do tamanho dos gânglios, e regressão das lesões ulceradas em face e clavícula. Na alta é orientado a tomar 2 comprimidos de Sulfametoxazol + Trimetropin de 8/8horas até reavaliação no ambulatório. Retorna no ambulatório escola de infectologia após um mês com regressão praticamente total da maior lesão (em região da clavícula) e melhora dos gânglios mantendo permanência apenas de gânglio em região submandibular.

\section{DISCUSSÃO}

O presente relato está de acordo com os dados da literatura que indicam maior incidência da PBmicose na população do gênero masculino e com idade aproximada de 20 anos e que residam em área rural (MESQUITA PMF, et al., 2016; ROSARIO JS, et al., 2017; MACEDO PM, et al., 2016; RADISIC MV, et al., 2017; MILLINGTON MA, et al., 2018). Nosso paciente apresentava lesão bucal na transição do palato para amígdala indo de encontro aos relatos já encontrados na literatura nas quais as principais lesões eram no rebordo alveolar da gengiva e no palato (MESQUITA PMF, et al., 2016; ROSARIO JS, et al., 2017; SHIKANAI-YASUDA MA, et al., 2017; ROSARIO JS, et al., 2017; MELO JA ,et al., 2017; ALMEIDA FA, et al., 2016). 
A forma subaguda da paracoccidiomicose é responsável por quase um quarto dos casos e é mais frequente nos estados do Maranhão, Minas Gerais, Pará, Goiás e São Paulo. Esta forma de apresentação predomina em crianças, adolescentes e adultos jovens, raramente acontecendo após os 40 anos de idade. No presente relato o indivíduo era um adulto jovem, porém a região que o mesmo era proveniente não era considerada uma região endêmica (SHIKANAI-YASUDA MA, et al., 2017; ROSARIO JS, et al., 2017; MELO JA ,et al., 2017; MACEDO $\mathrm{PM}$, et al., 2016). Esta apresentação da doença é conhecida por apresentar uma rápida disseminação do fungo por vários órgãos e geralmente o diagnóstico é feito após poucas semanas dos sintomas. No presente relato o paciente iniciou com sintomas duas semanas antes da internação indo de acordo com os dados da literatura (SHIKANAI-YASUDA MA, et al., 2017).

Uma rápida disseminação por órgãos se dá através do envolvimento do sistema fagocítico - mononuclear acarretando uma linfonodomegalia generalizada que pode com o decorrer dos dias supurar. Além disso podem ocorrer hepatoesplenomegalia, manifestações do trato digestivo, lesões de pele ou mucosas e envolvimento ósseo. O acometimento pulmonar é raro. Sintomas inespecíficos como febre, perda de peso podem acompanhar o quadro (SHIKANAI-YASUDA MA, et al., 2017; GAETTLI-JJE, et al., 2016). Concordante com a literatura nosso paciente apresentava linfonodomegalia generalizada, mais importante em região da face, cervical e axilar. $O$ paciente também apresentava hepatoesplenomegalia e lesões cutâneas que foram as responsáveis por o mesmo procurar atendimento. O paciente refere que apresentou perda ponderal desde o início dos sintomas e hiporexia (MELO JA, et al., 2017).

Em relação as alterações laboratoriais que podem ocorrer é a presença de eosinofilia ao hemograma. Ela é relativamente comum ocorrendo em até $50 \%$ dos casos. No presente caso o paciente apresentava 882 linfócitos dando uma porcentagem de $7 \%$ sendo que o valor de referência é de $2-5 \%$, ou seja, apesar de discreta eosinofilia este fator concorda com a literatura (SHIKANAI-YASUDA MA, et al., 2017; ROSARIO JS, et al., 2017). Em uma primeira avaliação já podem ser notadas algumas alterações ao exame físico que podem sugerir a forma aguda/subaguda da doença. Linfonodos aumentados em várias cadeias linfáticas e lesões cutâneas facilmente identificadas além de hepatoesplenomegalia já devem fazer pensar se em tal diagnostico. Como já citado o paciente apresentava linfonodomegalia generalizada e hepatoesplenomegalia, fígado e baço a dois centímetros do rebordo costal (SHIKANAI-YASUDA MA, et al., 2017).

Em casos mais avançados pode ocorrer icterícia, ascite e edema periférico principalmente decorrente de hipoalbuminemia. O mesmo não apresentava nenhum destes sintomas. Envolvimento do sistema nervoso central são mais raros ainda nesta forma o que concorda com nosso relato já que o paciente se manteve consciente e orientado durante todo o período (SHIKANAI-YASUDA MA, et al., 2017). Das manifestações inespecíficas que podem acompanhar o quadro dor abdominal, diarreia e vômitos são bastante frequentes acompanhadas de febre e perda ponderal (SHIKANAI-YASUDA MA, et al., 2017; SILVA JF, et al., 2016). Exames laboratoriais e de imagem que podem ser solicitados :Hemograma completo e provas inflamatórias, função e lesão hepática e função renal. Radiografia de tórax (SHIKANAI-YASUDA MA, et al., 2017; SILVA JF, et al., 2016).

Exames de imagem mais específicos como ultrassonografia, tomografias e ressonância magnéticas são excelentes para verificarem acometimento generalizado, mas só devem ser realizados caso a suspeita clínica e laboratorial de acometimento de órgãos que não possam ser identificados no exame físico (SHIKANAI-YASUDA MA, et al., 2017; SILVA JF, et al., 2016). Os exames iniciais apontaram provas inflamatórias aumentadas (PCR), mas função hepática e renal preservadas. Radiografia de tórax sem alterações, sendo assim, como paciente não apresentava suspeita clínica de envolvimento de outros órgãos não foram solicitados exames adicionais.

\section{Diagnóstico diferencial}

Como toda patologia há diagnósticos diferenciais que devem ser afastados estes são: linfoma, leucemia, histoplasmose, leishmaniose visceral e mononucleose infecciosa. Desta forma em nosso caso foram realizadas sorologias para mononucleose, citomegalovírus e toxoplasmose (síndromes mono like). Todas as sorologias eram negativas (ROSARIO JS, et al., 2017). 
O padrão ouro para fechar o diagnóstico de paracoccidioidomicose é a presença de Paracoccidioides spp no exame a fresco de escarro ou no raspado das lesões ou então na biopsia de órgãos acometidos (ROSARIO JS, et al., 2017; MELO JA, et al., 2017). Como paciente apresentava lesões cutâneas e múltiplo gânglios foi optado por biópsias dos mesmos onde foi confirmado o diagnóstico.

\section{Tratamento}

P. brasiliensis e $P$. lutzii são organismos sensíveis a maioria dos antifúngicos sistêmicos e até os bacteriostáticos derivados da sulfanilamida são eficazes. Podem então ser usados no tratamento os derivados azólicos (cetoconazol, fluconazol, itraconazol, voriconazol, posaconazol e isavuconazol), derivados sulfamídicos (cotrimoxazol, sulfadiazina etc.), a anfotericina B (formulações em deoxicolato, complexo lipídico e lipossomal), para formas graves,e mesmo a terbinafina (SHIKANAI-YASUDA MA, et al., 2017; ROSARIO JS, et al., 2016; SHIHIKANAI-YASUDA MA, et al., 2017; PEÇANHA PM, et al., 2016).

Atualmente a droga de escolha para o tratamento de formas leves e moderadas é o itraconazol na dose de $200 \mathrm{mg}$ diárias com altas taxas de eficácia e segurança. O tratamento pode durar de 9 a 18 meses e o paciente é constantemente reavaliado para critérios de cura clínica, imunológicos e radiológicos a depender da avaliação inicial. As lesões de pele e mucosa regridem em torno de 30 dias após o início do tratamento, os linfonodos em cerca de 45 a 90 dias e a cura ou estabilização radiológica e vista após 6 meses de tratamento (SHIKANAIYASUDA MA, et al., 2017).

O paciente em questão foi avaliado ambulatorialmente 20 dias após o início do tratamento e sete dias após a alta e já apresentava regressão dos linfonodos com permanência ainda de linfonodo importante em região submandibular, regressão quase total das lesões cutâneas (SHIKANAI-YASUDA MA, et al., 2017).

Os pacientes com formas mais avançadas necessitam de medidas aliadas para um resgate do estado nutricional adequado. Além disso deve ser estimulado medidas não farmacológicas como controle do tabagismo e etilismo (SHIKANAI-YASUDA MA, et al., 2017). O paciente com Pbmicose necessita de um acompanhamento periódico, mas que pode ser realizado ambulatoriamente (SHIKANAI-YASUDA MA, et al., 2017; MELO JA, et al., 2017).

Nesta patologia não podemos usar a expressão cura definitiva já que é impossível a erradicação do fungo do organismo. É possível alcançar níveis baixos de carga fúngica no organismo permitindo que a imunidade celular seja recuperada. É recomendado que após o estabelecimento de cura sejam mantidos exames periódicos por até um ano (SHIKANAI-YASUDA MA, et al., 2017; MELO JA, et al., 2017).

Ainda não está disponível uma vacina para prevenção desta doença e a maneira de prevenção é evitando a exposição a poeira de escavação do solo e uso de ferramentas de proteção individual no momento de manipulação do solo, principalmente na colheita de café (SHIKANAI-YASUDA MA, et al., 2017).

$\mathrm{APb}$ micose é uma micose profunda de grande importância epidemiológica ainda nos dias de hoje. No entanto, a mesma por não ser de notificação compulsória é ainda subdiagnosticada. É uma patologia que deve ser sempre lembrada em casos de linfonodomegalia generalizada e hepatoesplenomegalia. Seu diagnóstico envolve fatores clínicos, laboratoriais e biópsia. Seu tratamento envolve drogas de fácil acesso como o sulfametoxazol + trimetoprim e pode ser realizado em ambiente ambulatorial.

\section{REFERÊNCIAS}

1. ALMEIDAFA, et al. Paracoccidioidomycosis in Brazilian patients with and without human immunodeficiency vírus infection. Am J Trop Med Hyg, 2016,96(2):368-375.

2. GAETTLI-JJE, et al. Etiologia, epidemiologia e manifestações clínicas da paracoccidioidomicose. Archives of Health Investigation, 2016; 5(2): 98-105.

3. MACEDO PM, et al. Paracoccidioides brasiliensis PS2: first autochthonous paracoccidioidomycosis case report in Rio de Janeiro, Brazil, and literature review. Mycopathologia, 2016 ,181(9-10):701-800. 
4. MELO JA, et al. Paracoccidodomicose : descrição das espécies e morbidade da doença, Journal Of medicine and health promotion, 2017,801-809.

5. MESQUITA PMF, et al. Paracoccidioidomicose brasiliensis em sistema nervoso central. Arquivos Brasileiros de Neurocirurgia: Brazilian Neurosurgery, 2016.

6. MILLINGTON MA, et al. Paracoccidioidomicose: abordagem histórica e perspectivas de implantação da vigilância e controle. Epidemiol. Serv. Saúde, 2018.

7. MUNÕZ JF, et al. Genome diversity, recombination, and virulence across the major lineages of Paracoccidioides. MSphere, 2016.

8. PEÇANHA PM, et al. Amphotericin B lipid complex in the treatment of severe paracoccidioidomycosis: a case series. Int J Antmicrobiol Agents, $2016 ; 48(4): 428-30$.

9. RADISIC MV, et al. Acute pulmonary involvement by paracoccidioidomycosis disease immediately after kidney transplantation: case report and literature review. Transpl Infect Dis, 2017; 19(2).

10. ROSARIO JS, et al.Diagnóstico e tratamento de paracoccidiomicose: relato de caso clinico. Revista eletrônica UNIVAP, 2017; 22(40).

11. SHIHIKANAI-YASUDA MA, et al. International Society for Human and Animal Mycology. Working group on Paracoccidioidomycosis, 2017.

12. SHIKANAI-YASUDA MA, et al. Brazilian guidelines for the clinical management of Paracoccidiodomycosis. Rev Soc Bras Med Trop, 2017;50(5):715-800.

13. SILVA JF, OLIVERIA HC, et al. Advances and challenges in paracoccidioidomycosis serology caused by Paracoccidioides species complex: an update. Diagc Microbiol Infect Dis, 2016; 84(1):87-94.

14. TOZETTO-MENDOZA TR, et al. Role of T. cruzi exposure in the pattern of $\mathrm{T}$ cell cytokines among chronically infected HIV and Chagas disease patients. Clinics. In press, 2017.

15. VALLE ACF, et al. Paracoccidioidomycosis after highway construction, Rio de Janeiro, Brazil. Emerg Infect Dis, 2017;23(11):1917-1930.

16. VOGELMEIER CF, et al. Global strategy for the diagnosis, management, and prevention of chronic obstructive lung disease 2017 report. GOLD executive summary. Am J Respir Crit Care Med, 2017;195(5):557-570. 